\title{
The value of weather radar data for the estimation of design storms - an analysis for the Hannover region
}

\author{
Uwe Haberlandt and Christian Berndt \\ Institute of Water Resources Management, Hydrology and Agricultural Hydraulic Engineering, \\ Leibniz University of Hannover, Hannover, Germany \\ Correspondence to: Uwe Haberlandt (haberlandt@iww.uni-hannover.de)
}

Published: 12 May 2016

\begin{abstract}
Pure radar rainfall, station rainfall and radar-station merging products are analysed regarding extreme rainfall frequencies with durations from $5 \mathrm{~min}$ to $6 \mathrm{~h}$ and return periods from 1 year to 30 years. Partial duration series of the extremes are derived from the data and probability distributions are fitted. The performance of the design rainfall estimates is assessed based on cross validations for observed station points, which are used as reference. For design rainfall estimation using the pure radar data, the pixel value at the station location is taken; for the merging products, spatial interpolation methods are applied. The results show, that pure radar data are not suitable for the estimation of extremes. They usually lead to an overestimation compared to the observations, which is opposite to the usual behaviour of the radar rainfall. The merging products between radar and station data on the other hand lead usually to an underestimation. They can only outperform the station observations for longer durations. The main problem for a good estimation of extremes seems to be the poor radar data quality.
\end{abstract}

\section{Introduction}

Design storms are required for the planning and evaluation of hydraulic structures and flood risk management in urban and rural catchments. The design storms are derived from frequency analyses of annual maximum rainfall or rainfall above a threshold for specific durations. The storms are usually condensed for different durations and frequencies to intensity-duration-frequency (IDF) curves or depth-durationfrequency curves (DDF) for a certain location. In order to obtain reliable estimation of design rainfall, long-term precipitation observations in high temporal resolution are required. Especially short duration observations are often only available with poor spatial density, which demands regionalisation. There have been different studies about regionalisation of DDF curves over the last years (Durrans and Kirby, 2004; Johnson et al., 2016; Madsen et al., 2002). Also scaling methods have been applied to derive IDF curves for short durations from better available daily observations (Yu et al., 2004).

An alternative would be to use weather radar for the estimation of design rainfall, which is available in a high spatial and temporal resolution, or at least to use it as an addi- tional information for regionalisation. Meanwhile the observation length of many operational radar instruments extend over a time period of 10 years, which suggests to analyse their benefits for estimating design rainfall. Rainfall derived from radar data is usually biased and needs some kind of correction. This can be done by adjusting the radar data (Krajewski and Smith, 2002) or by merging radar data and observations (Berndt et al., 2014). So far, only a few investigations have been carried out utilising radar rainfall for extreme value analyses. Marra and Morin (2015) used a 23-year radar record to estimate IDF curves for different climatic zones in Israel. They found a general overestimation of radar based rainfall extremes compared to the gauge data, but with $70 \%$ of the cases within the uncertainty bounds of the rain gauge derived IDF's. Eldardiry et al. (2015) analysed the contribution of different factors to the uncertainty in the estimation of design storms. They employed a 13-year data set from the NEXRAD radar network for the Louisiana region in the USA and found that radar data underestimate the observed gauge based IDF curves due to the conditional bias of the radar product. They also found that a regional estimation of the IDF curves, e.g. using the index flood method, reduces 
the uncertainty significantly compared to the at site estimations. Overeem et al. (2009) used a 11-year radar data set for extreme value analyses in the Netherlands. They found that the radar data are suitable for the estimation of DDF curves if regional frequency analyses is applied. However, the uncertainty for the estimation of storms with longer durations becomes large due to the short sample and stronger spatial correlation of events.

In the current study, different regionalisation methods are compared to estimate DDF curves from interpolated rainfall products with and without utilising radar information. This is supposed to provide insights about the real benefit of radar data for at site estimation of DDF curves compared to using gauge based rainfall data only.

\section{Methodology}

\subsection{Radar data pre-processing}

The radar data pre-processing was performed according to Berndt et al. (2014). In the following, those steps are briefly summarized: (1) raw radar reflectivities at 5 min resolution were transformed into rainfall intensities using a standard $Z-R$ relationship; (2) a simple statistical clutter correction method was applied; (3) the data were interpolated on a $1 \mathrm{~km} \times 1 \mathrm{~km}$ grid; (4) a space-time filter was applied on this grid for smoothing; (5) outliers were removed considering the cumulative distribution function of standard errors between rain gauge and radar data. In addition, different approaches for radar data adjustment to gauge data are employed, which are described together with the interpolation methods below.

\subsection{Rainfall estimation for unobserved locations}

Continuous 5 min point precipitation time series are estimated for a set of locations for which observed rainfall is available, however without using the observations at the target location in the estimation procedure; i.e. a cross- validation is performed. Thus, a real validation of the estimation method is possible assuming that the observations are error free. The following methods are used for the estimation/interpolation of rainfall data sets:

1. REF - This represents the observed reference rainfall time series, which is taken without modification.

2. NN-A nearest neighbour interpolation using recording rainfall stations is carried out.

3. OK - Ordinary kriging is applied using the $m$ closest surrounding recording rainfall stations.

4. Radar-Pre-processed radar data as described above are extracted from the nearest $1 \mathrm{~km} \times 1 \mathrm{~km}$ pixel and taken without further adjustment.
5. RadarADJ - Radar data are adjusted with daily rainfall using the denser network of non-recording stations.

6. $\mathrm{CM}$ - Conditional merging interpolation (Sinclair and Pegram, 2005) is applied using data from recording stations and radar data without adjustment (Radar).

CMADJ - Conditional merging interpolation is applied using data from recording stations and radar with adjustment (RadarADJ).

For performance assessment the relative bias

$r$ bias $=\frac{1}{S \cdot R} \sum_{i=1}^{S} \sum_{j=1}^{R}\left(\frac{\hat{y}_{i, j}-y_{i, j}}{y_{i, j}}\right)$

and the relative root mean squared error

$r$ rmse $=\sqrt{\frac{1}{S \cdot R} \sum_{i=1}^{S} \sum_{j=1}^{R}\left(\frac{\hat{y}_{i, j}-y_{i, j}}{y_{i, j}}\right)^{2}}$

are used, were $\hat{y}$ and $y$ are the estimated and observed design storm quantiles, respectively, and $S$ and $R$ are the number of stations and return periods, respectively. The calculations are carried out separately for different storm durations $D$.

\subsection{Extreme value analyses}

Basis for the extreme value analyses are the 5 min time series obtained from the interpolation methods listed above. The extreme value analysis is carried out according to the German standards for design storm estimation (DWA-M-531, 2012). Partial duration series (PDS) are built with a sample size of about $e$ times the number of years for durations with $D=5$, $10,15,30,60,120,240,360,720,1440 \mathrm{~min}$. A minimum dry spell duration of $\operatorname{Min}[4 \mathrm{~h}, D]$ is applied to guarantee independence of the storms. The exponential probability distribution is fitted to the PDS for each duration. Finally, the parameters of the distributions are smoothed over the durations to allow a consistent estimation of DDF curves without jumps (see DWA-M-531, 2012).

\section{Study area and data}

The study area is the radar range with a radius of $128 \mathrm{~km}$ for the weather radar at the Hannover airport (see Fig. 1). This region has elevations from the sea level in the northern mostly flat part up to $1141 \mathrm{~m}$ a.s.l. in the Harz Mountains in the South. The average annual precipitation varies between 500 and $1700 \mathrm{~mm} \mathrm{yr}^{-1}$. Radar data were available for the period from 2000 to 2012 (13 years). The radar data preprocessing was carried out as explained in Sect. 2.1. Eight recording reference stations have been selected for which a validation of the DDF curves was carried out. In addition, 46 recording stations and 512 non-recording stations were 


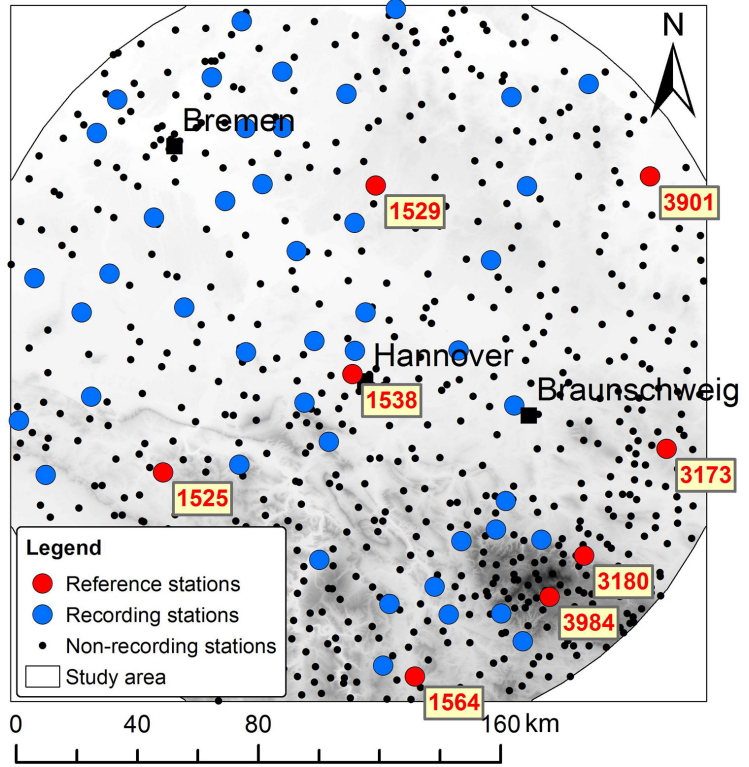

Figure 1. Study area with topography, radar circle and rainfall stations.

available for the interpolations within the study area, however with highly varying temporal coverage. For all stations, the same 13 years period as for the radar observations was used.

\section{Results}

As a starting point, the estimation of mean annual precipitation using the different interpolation methods is evaluated. Figure 2 shows a comparison of the mean annual precipitation for the 13 years averaged over the 8 reference stations using the different methods. The two approaches, which do not use radar data, $\mathrm{NN}$ and $\mathrm{OK}$, provide interpolated time series almost without bias. Employing pure radar for the estimation leads to a significant underestimation, which is typical for comparisons between radar rainfall and station values. When radar data are adjusted with rainfall from daily stations, the bias in the annual values is removed. The interpolations using conditional merging with radar data (CM) and with adjusted radar data (CMADJ), respectively, lead to slight overestimations here.

In terms of the extremes, the results are different from the mean values. Figure 3 shows the relative bias for the design rainfall estimation using the different interpolation methods averaged over the 8 reference stations and 8 return periods $T=1,2,3,5,10,20,25$ and 33 years. The relative bias using the pure radar data (Radar) reveals a huge overestimation of the extremes. Comparing the selected events from pure radar data and station data for the same locations shows only little temporal overlap. This indicates that there might be still considerable errors and outliers in the radar data, which

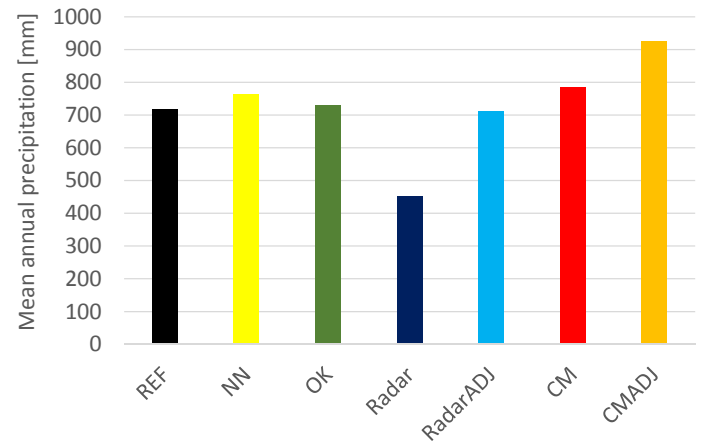

Figure 2. Mean annual rainfall from 5 min interpolated rainfall series for the period 2000 to 2012 averaged over the 8 reference stations.

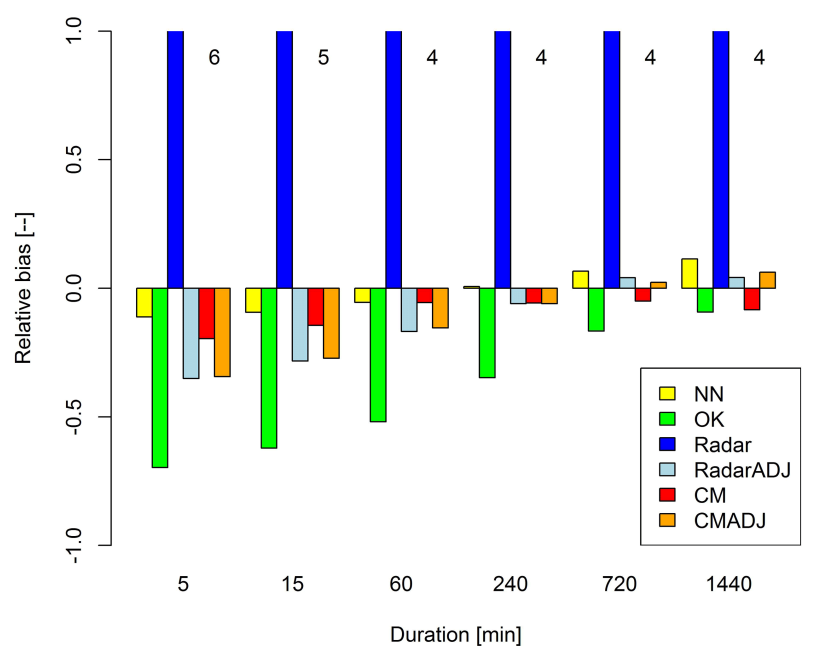

Figure 3. Relative bias averaged over the 8 reference stations and 8 return periods ( $T=1,2,3,5,10,20,25,33$ years).

do not represent real rainfall. Ordinary kriging (OK), which does not include radar data, shows the largest negative bias. This is likely due to the strong smoothing behaviour of this method. The smallest bias is obtained for the simple nearest neighbour (NN) interpolation. Also acceptable is the bias for the conditional merging $(\mathrm{CM})$ technique without radar data adjustment. The methods with daily adjusted radar rainfall, RadarADJ and CMADJ, still express significant biases for short durations.

Figure 4 shows a comparison of the relative root mean squared errors obtained from the different methods, again averaged of the 8 reference stations and 8 return periods. Using pure radar data (Radar) produces the largest error followed by $\mathrm{OK}$, which uses only the recording stations. The overall smallest error is found when applying the simple nearest neighbour technique (NN) for interpolation. The second smallest error is obtained when conditional merging (CM) is used, which can outperform the NN approach only for longer durations. The methods with daily adjustments, RadarADJ 


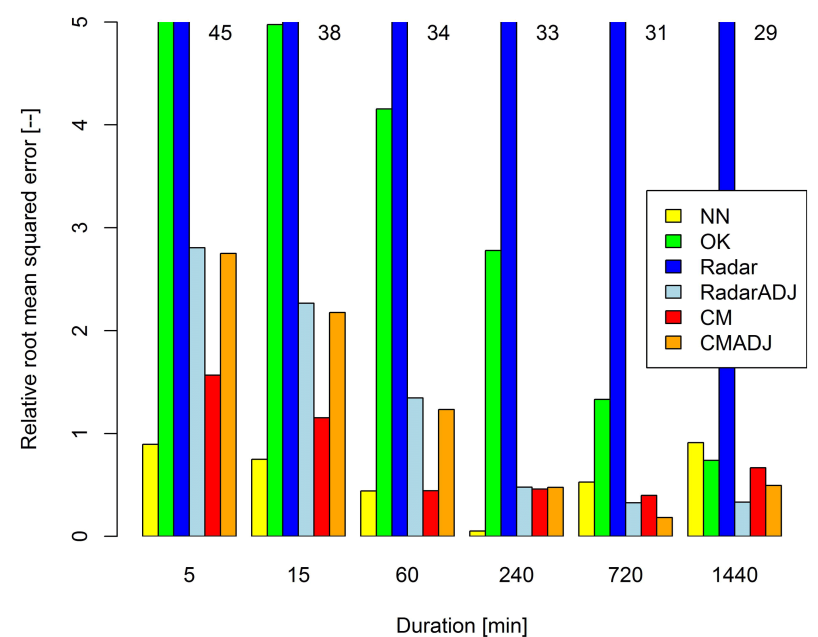

Figure 4. Relative rout mean squared error averaged over the 8 reference stations and 8 return periods $(T=$ $1,2,3,5,10,20,25,33$ years).

and CMADJ, have still considerable high errors for short rainfall durations.

In Fig. 5 exemplarily estimated DDF curves for the rainfall station Hannover are presented for return periods $T=1$, 5,10 and 20 years. The pure radar DDF is only visible for $T=1$ years and outside the $x$ axes range for the other return periods. This shows again the large overestimation if pure radar data are used to estimate the extremes. The errors for the estimation of the DDF curves increase with increasing return period. The DDF curves based on OK show again a significant underestimation. The best methods for the station Hannover is CMADJ. The best method varies between the stations with an average performance as indicated in Fig. 4.

\section{Summary and conclusions}

This study has investigated the benefit of radar data for the estimation of design rainfall. Different interpolation methods were applied on $5 \mathrm{~min}$ time series from recording rain stations, radar data and merging products. The interpolated data sets were used for extreme value analyses and the estimation performance was assessed based on observations. The results and conclusions from this analysis can be summarized as follows:

- Using pure radar data leads to large overestimation of DDF curves. This is probably due to measurement errors for the weather radar, which could not be detected and corrected in pre-processing.

- The nearest neighbour approach gives overall the best results. This is partly due to the non-smoothing character of this method and due to the sufficiently dense network of recording rainfall stations.
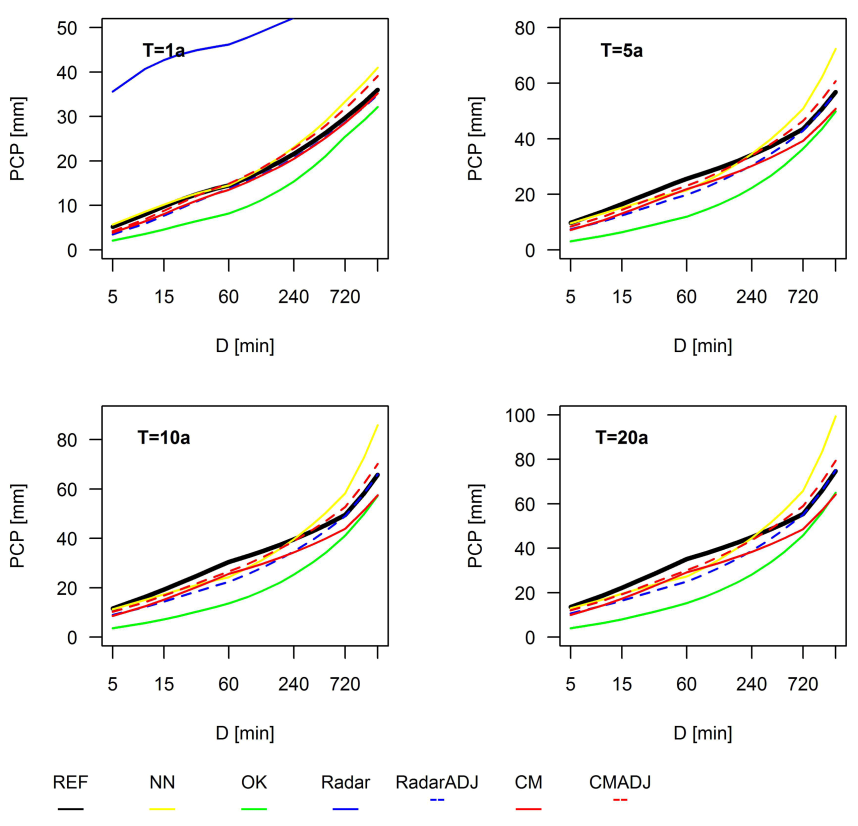

Figure 5. Depth duration frequency curves for the station Hannover (id 1538 in Fig. 1) for four selected return periods $T=$ $1,2,10,20$ years.

- The radar-gauge merging methods reduce the error considerably but are best only for long durations. However, they all provide a negative bias, which can be explained by the smoothing effect of the interpolation methods.

- Ordinary kriging leads to the strongest underestimation of the design storms due to these smoothing effects. This approach cannot be recommended when extreme value analysis is required.

Overall, the results indicate that using radar data in this manner will not benefit the estimation of design rainfall, although this might be different for other study regions and radar instruments. Anyway, for improvements it is necessary to better correct the radar data for errors. A regional frequency analysis of extreme rainfall using methods similar like the index flood method might lead to better results. In addition, a procedure, which first estimates extreme value distributions locally and then interpolates the parameters in space, might be better for avoiding negative biases.

Acknowledgements. We thank the German Weather Service (DWD) for providing the precipitation and radar data. 


\section{References}

Berndt, C., Rabiei, E., and Haberlandt, U.: Geostatistical merging of rain gauge and radar data for high temporal resolutions and various station density scenarios, J. Hydrol., 508, 88-101, 2014.

Durrans, S. R. and Kirby, J. T.: Regionalization of extreme precipitation estimates for the Alabama rainfall atlas, J. Hydrol., 295, 101-107, 2004.

DWA-M-531: Merkblatt: Starkregen in Abhängigkeit von Wiederkehrzeit und Dauer, Deutsche Vereinigung für Wasserwirtschaft, Abwasser und Abfall e. V., Hennef, 2012.

Eldardiry, H., Habib, E., and Zhang, Y.: On the use of radar-based quantitative precipitation estimates for precipitation frequency analysis, Part 2, J. Hydrol., 531, 441-453, 2015.

Johnson, F., Hutchinson, M. F., The, C., Beesley, C., and Green, J.: Topographic relationships for design rainfalls over Australia, J. Hydrol., 533, 439-451, 2016.

Krajewski, W. F. and Smith, J. A.: Radar Hydrology: rainfall estimation, Adv. Water Resour., 25, 1387-1394, 2002.
Madsen, H., Mikkelsen, P. S., Rosbjerg, D., and Harremoës, P.: Regional estimation of rainfall intensity-duration-frequency curves using generalized least squares regression of partial duration series statistics, Water Resour. Res., 38, 1239, doi:10.1029/2001WR001125, 2002.

Marra, F. and Morin, E.: Use of radar QPE for the derivation of Intensity-Duration-Frequency curves in a range of climatic regimes, Part 2, J. Hydrol., 531, 427-440, 2015.

Overeem, A., Buishand, T. A., and Holleman, I.: Extreme rainfall analysis and estimation of depth-duration-frequency curves using weather radar, Water Resour. Res., 45, W10424, doi:10.1029/2009wr007869, 2009.

Sinclair, S. and Pegram, G.: Combining radar and rain gauge rainfall estimates using conditional merging, Atmos. Sci. Lett., 6, 19-22, 2005.

Yu, P.-S., Yang, T.-C., and Lin, C.-S.: Regional rainfall intensity formulas based on scaling property of rainfall, J. Hydrol., 295, 108-123, 2004. 